MONIKA SCHÖNHERR

\title{
Finitheit und Infinitheit: Routinen im Bereich der Kodierung verbaler Prädikationen aus diachroner und typologischer Sicht
}

\begin{abstract}
Celem niniejszej pracy jest zbadanie syntaktycznych strategii eliminacji predykatów czasownikowych ze struktur zdaniowych w różnych konstrukcjach na przykładzie starogermańskich systemów językowych. Analizowane będą przypadki, w których dany predykat nie jest formalnie kodowany i w których na skutek tego dochodzi do powstania tzw. predykacji ukrytej. Planowane badanie będzie miało dwa cele: Po pierwsze podjęta będzie próba wyróżnienia poszczególnych typów struktur zawierających predykację ukrytą w dwóch typologicznie różnych obszarach językowych: w języku (staro-wysoko-)niemieckim i języku gockim jako językach germańskich oraz w języku polskim jako języku słowiańskim. Drugie zadanie polegać będzie na analizie struktur zawierających predykację ukrytą pod kątem ich właściwości składniowych i semantycznych.
\end{abstract}

Ziel des vorliegenden Beitrags ist es, die syntaktischen Strategien der Auslassung der finiten Verben in verschiedenen Konstruktionen in der Altgermania zu untersuchen. Analysiert werden Fälle, in denen das verbale Finitum auf der Ausdrucksseite formal nicht kodiert wird, sodass es zur Entstehung der sog. koverten Prädikation kommt. Die geplante Untersuchung verfolgt insgesamt zwei Ziele: Erstens werden die einzelnen Strukturen mit der koverten Prädikation in zwei typologisch unterschiedlichen Sprachbereichen (dem Althochdeutschen und Gotischen als germanische Sprachen einerseits, dem Polnischen als Vertreter des slawischen Sprachareals andererseits) differenziert. Zweitens zielt die Studie darauf ab, die Konstruktionen mit koverter Prädikation hinsichtlich ihrer semantischen und syntaktischen Eigenschaften zu untersuchen.

In this paper I examine the syntactic strategies of omitting the finite verbs in various constructions across the Germanic languages. I explore cases in which the verbs are formally not marked and the so-called covert predication is established. The study will 
have two aims: Firstly, I try to group individual types of the structures with the covert predication in two typologically distinct language areas: in (Old-High-)German and Gothic as Germanic languages, on the one hand, and Polish as a Slavic language, on the other hand. Secondly, I will investigate the syntactic properties and the semantic components of the non-finite/covert structures.

\section{Einführende Bemerkungen}

Die Kodierung verbaler Satzpropositionen erfolgt prototypisch durch das verbale Prädikat, das ausdrucksseitig als finite Verbform erscheint und den strukturell-semantischen Satzkern konstituiert. Während der verbozentrische Mechanismus als eine Routine im Bereich der Kodierung von Prädikationen angesehen werden kann, gelten die ,weniger finiten“1 oder die nicht-finiten Prädikationsmuster als randständige Erscheinungen, die in den klassischen grammatischen Abhandlungen nicht speziell untersucht oder zugunsten der verbozentrischen Satzkonstruktionen aus dem Zentrum des syntaktischen Interesses gerückt werden. Die Untersuchungen am authentischen Sprachmaterial beweisen jedoch, dass nicht-finit kodierte Satzprädikationen eine durchaus reguläre Distribution aufweisen und keineswegs als ein Randphänomen zu beurteilen sind. Aus der Sicht der Pragmatik und Textgrammatik ist die In- bzw. Afinitheit sehr stark an bestimmte Textsorten gebunden. So wird z. B. in Texten mit abgeschwächter kommunikativer Ausrichtung (z.B. Tagebuchtexte) nicht so stark auf die Finitheit geachtet wie z. B. in narrativen Texten. Andererseits kann aber Nicht-Finitheit auch umgekehrt zur stärkeren kommunikativen Geltung eines Textes beitragen (vgl. Stileffekte bei EROMS 2008), die durch bewusste Auslassung des Verbums finitum (etwa in den Werbetexten) entstehen. Ob die infniten Prädikationsmuster denselben grammatischen Status wie finite Satzstrukturen haben, ist in der einschlägigen Literatur nicht endgültig geklärt. ${ }^{2}$ Die meisten Grammatikkonzepte gehen davon aus, dass der Satz stets eine formbezoge bestimmte Einheit ist, in der

1 Givón (1990) geht davon aus, dass Finitheit ein skalares Phänomen ist, bei dem zwischen unterschiedlich stark finiten (most finit - least finit) Konstruktionen differenziert werden kann. Partizipial-, Infinitiv und Nominalstrukturen lassen sich an der Finitheitskala im unteren Bereich ansiedeln - als Konstruktionen mit dem am schwächsten ausgeprägten Finitheitswert.

2 ENGel (1996:180) setzt z.B. das Vorhandensein eines finiten Verbs als oberstes Kriterium für einen Satz an, an einer anderen Stelle (ENGEL 1994:152) behandelt er verblose Konstruktionen des Typs Feuer! als „Kurzsätze“ [Hervorhebung M.S.]. 
ein finites Verb und die dazugehörigen Komplemente enthalten $\operatorname{sind}^{3}$ (vgl. z. B. ZIFONUN 1997:86f.). Die Frage, ob dem Kriterium der Verbzentriertheit bzw. der Finitheit dieselbe Relevanz auch in der Diachronie und in der Panchronie zugesprochen werden kann, ist ebenfalls weitgehend offen.

Im Folgenden behandle ich die nicht-finiten Konstruktionen - ähnlich wie ihre finiten Pendants - als Sätze und das Vorhandensein einer Prädikation in einem Syntagma - als das wichtigste satzkonstituierende Kriterium. ${ }^{4}$ Gerade in typologischer Sicht gibt es keine schwerwiegenden Gründe, eine trennscharfe Grenze zwischen Syntagmen mit overt realisierter Finitheit und Syntagmen, die kein finites Verb an der terminativen Satzoberfläche enthalten, zu ziehen. Beide Konstruktionstypen können ohne weiteres unter dem Oberbegriff ,Satz' subsumiert werden. Das ,Genus proximum', das beide Konstruktionstypen vereint, ist die Prädikation. So gesehen kann zwischen Sätzen mit overter Prädikation, die durch ein finites Verb auf der Ausdrucksseite gekennzeichnet sind, und solchen, die durch das Auslassen eines expliziten Verbums finitum einen impliziten bzw. koverten Zugriff auf die Satzproposition ermöglichen, differenziert werden.

Die Aufgabe dieses Beitrags besteht nun u. a. darin, dem Verhältnis von Finitheit und Nicht-Finitheit im diachronischen und typologischen Vergleich nachzugehen. Die Analyse zielt darauf ab zu zeigen, welche Typen von infiniten Satzmustern in den untersuchten Korpustexten ausdifferenziert werden können und wie sie semantisch und syntaktisch in die gesamte Satzstruktur eingebettet sind. Die jeweiligen Analyseschritte schlagen sich in der Struktur der weiteren Ausführungen nieder. In Kapitel 2 werden zunächst methodische Fragen zur Syntaxforschung der historischen Sprachstufen behandelt. Daraufhin werden Überlegungen zu Finitheit und Nicht-Finitheit (Kapitel 3) sowie Prädikation (Kapitel 4) als die zentralen Begriffe des vorliegenden Artikels angestellt. Das Kapitel 5 enthält eine Analyse historischer und moderner Sprachbelege, die unterschiedliche Kodierungstechniken koverter Satzprädikationen aus diachroner und kontrastiver Sicht präsentieren. In dem zusammenfassenden Kapitel werden die wichtigsten Schlussfolgerungen aus den vorstehenden Kapiteln rekapitulierend dargestellt. Die empirische Unter-

3 Daneben gibt es auch funktional bestimmte kommunikative Einheiten, mit denen grundlegende sprachliche Handlungen (wie etwa Informieren, Fragen, Befehlen) vollzogen werden können, die jedoch nicht den Status eines Satzes haben (vgl. ZIFONUN 1997:86f.).

4 Mein Ziel ist nicht, die bisher ausgearbeiteten Satzkonzepte zu schmälern, sondern diese um die universal-typologische Perspektive zu erweitern. 
suchungsbasis bilden typologisch unterschiedliche Sprachsysteme, damit möglichst unterschiedliche Typen der koverten/impliziten Prädikationsmuster zur Geltung kommen können. Obwohl sich die Reihenfolge der dargestellten Belege grundsätzlich nach dem diachronen Kriterium richtet, geht es vielmehr um das Aufdecken universal-typologischer Mechanismen im Bereich der Kodierung verbaler Prädikationen. Die Analyse der Belege ist intra- und interlingual ausgerichtet: Der intralinguale Vergleich (Althochdeutsch deutsche Gegenwartssprache) soll zeigen, welche Mechanismen der Finitheitsmarkierung in den zeitlich am weitesten auseinanderliegenden Sprachzuständen vorkommen, um dadurch möglichst kontrastreiche und differenzierte Finitheitsmuster zu erhalten. Bewusst wurde auf die Zwischenstufen des Mittelhochdeutschen und Frühneuhochdeutschen verzichtet, da in der vorliegenden Arbeit keine historisch-diachrone, die jeweiligen Entwicklungsetappen der Finitheit umfassende Studie angestrebt wird, sondern vielmehr eine universal-typologisch begründete Herangehensweise im Vordergrund steht. Auch die Stichproben aus den beiden Vergleichssprachen (Gotisch und Polnisch) werden hier lediglich zur interlingualen Kontrastierung der Finitheitsmuster aufgeführt, keinesfalls zur Ermittlung von Parallelentwicklungen im Bereich des behandelten Problemkomplexes. ${ }^{5}$ Die Untersuchungsbasis bilden altgermanische Bibeltexte (Gotische Bibel aus dem 4. Jahrhundert, das althochdeutsche Tatian aus dem 9. Jahrhundert). Für das Polnische und die deutsche Gegenwartssprache werden entsprechend Belege aus dem polnischen und neuhochdeutschen Text der Bibel ausgewertet.

\section{Methodische Überlegungen zur historischen Syntaxforschung}

Bei der Untersuchung der altgermanischen Satzstrukturen gibt es eine Reihe schwer überwindbarer Hindernisse, die eine adäquate empirische Erforschung der syntaktischen Hauptphänomene wie etwa Topologie, Satzmuster, Satzmodelle und/oder Satzgrenzen erschweren. Dieser Umstand ist u. a. darauf zurückzuführen, dass es sich bei den altgermanischen, darunter den gotischen und althochdeutschen Prosatexten fast durchweg um Übersetzungstex-

5 Die Ergänzung der Untersuchungsperspektive um eine historisch-diachrone Studie mit Längsschnittanalysen auf der jeweiligen Sprachstufe des Deutschen wird im Rahmen einer weiteren Arbeit geplant. Auch die Einbeziehung von Parallelkorpora aus dem Bereich der Altgermania (Altenglisch, Altisländisch etc.) und der Altslavia (Altpolnisch) bildet einen der Schwerpunkte der nachfolgenden Untersuchungen. 
te handelt, die typischerweise die syntaktischen Muster der (griechischen oder lateinischen) Originalvorlage mehr oder weniger nachbilden (vgl. hierzu SCHÖNHERR 2012:126f.). So ist z.B. bei der lateinisch-althochdeutschen Tatianbilingue davon auszugehen, dass viele syntaktische Strukturen im deutschen Text keine originären bzw. genuinen Konstruktionen darstellen, ja stellenweise völlig untypisch oder unauthentisch wirken (vgl. FLEISCHER 2006:31f.). Vergleichbares gilt auch für das Gotische: Abgesehen von den rein quantitativen Defiziten der Überlieferungssituation des Gotischen (außer der Wulfila-Bibel, die auf eine griechische Vorlage zurückgeht, sind keine größeren autochthonen Texte erhalten geblieben) müssen die Belege und mithin deren Aussagekraft über die Sprachwandelprozesse bzw. die Entwicklungstendenzen im Bereich der topologisch-syntaktischen Strukturen stets vor dem Hintergrund des fremdsprachigen Einflusses seitens der griechischen Syntax betrachtet werden. Demgegenüber umfasst die althochdeutsche Schriftperiode eine Reihe von autochthonen, jedoch metrisch und reimgebundenen Texten, die zwar inhaltlich und sprachlich weitgehend autonom sind, sich wegen ihrer Reimform aber nur bedingt für die Untersuchung der topologisch-syntaktischen Fragestellungen eignen. Die Bildung von syntaktischen Strukturen folgt dabei keinen klaren satzstrukturellen Normen, sondern ist weitgehend durch Erfordernisse der äußeren Textform gesteuert. Das Paradebeispiel dafür ist der Text des Hildebrandsliedes, in dem die versrhythmischen Gesetzmäßigkeiten, allen voran die Alliteration, den syntaktischen Kriterien des Satzbaus übergeordnet sind (vgl. KoTIN 2007:240):

4 sunufatarungo iro saro rihtun,

,Sohn und Vater ihre Rüstung richteten

${ }_{5}$ garutun se iro gudhamun, gurtun sih iro suert ana,

,bereiteten sie ihre Kampfkleider, gürteten sich ihre Schwerter um, ‘

${ }_{6}$ helidos, ubar hringa, do sie to dero hiltiu ritun.

,die Helden, über Ringgewänder, als sie zum Kampf ritten

Die Stellung der Satzglieder in dem kurzen Textstück kann auf eine dreifache Alliteration zurückgeführt werden: Die Alliteration auf $s$ in Vers 4 hat ihre ikonische Entsprechung in topologischer Nähe vom Satzsubjekt und -objekt; die Alliteration auf $g$ ergibt in Zeile 5 die VSO/VO-Folge und in Vers 6 liegt aufgrund der Alliteration auf $h$ eine - heute - ungewöhnliche Serialisierung der Satzentitäten vor. Die Syntagmen in diesem kurzen Text sind also keine nach einem bestimmten Satzbauplan konstruierten Sätze mit klar umrissenen Grenzen und Strukturen. Vielmehr lassen sie sich als aneinandergereihte, semantisch abhängige Prädikationen-Sequenzen einordnen (vgl. GvOZDECK- 
AJA 2002:143f.; zit. nach KoTIN 2007:240) - ein Merkmal, das sie, wie aus dem Weiteren ersichtlich wird, doch als Sätze behandeln lässt.

Nichtsdestoweniger sind die Erforschung der historischen Sprachdaten und die Suche nach möglichst weiträumig gültigen Aussagen über die Satzstrukturierung in der Altgermania sowie deren gemeinsame Mechanismen und Kriterien durchaus möglich. Dabei empfiehlt es sich, einen Abgleich der aus unterschiedlich konzipierten (Prosa- und Vers-)Texten stammenden Parallelbelege und ihnen zu Grunde liegenden (semantischen, strukturellen etc.) Gemeinsamkeiten vorzunehmen. Am aussagekräftigsten für die Untersuchung historischer Syntaxphänomene, darunter des hier behandelten Phänomens, sind die sogenannten Kontrastbelege, d.h. die Textstellen, die syntaktisch von der Originalvorlage differieren und auf genuine Entwicklungstendenzen hinweisen.

\section{Finitheit - Nichtfinitheit}

Die Kategorie der Finitheit gehört zu den ältesten, bereits in der antiken Grammatiktradition etablierten grammatischen Kategorien. Seit Beginn des Nachdenkens über Sprache wurden die sprachlichen Formen entweder als finit, d.h. auf einen konkreten Fall, ein Objekt bzw. eine Handlung beschränkt, oder als nicht-finit, d.h. auf Eigenschaften von irgendwelchen Entitäten bezogen, aufgefasst. ${ }^{6}$ Demzufolge referiert jede finite Sprachform auf ein durch bestimmte Merkmale markiertes Phänomen oder eine Menge von Phänomenen, weswegen der Finitheitsbegriff semantisch in die Nähe von Determination rückt. Wissenschaftsgeschichtlich war die Distinktion zwischen finiten (durch „limiting features“ begrenzten) und infiniten (durch „limiting features" nicht begrenzten) Formen zunächst im Bereich der Nomina von Bedeutung (vgl. NIKOLAEVA 2007:1). Der Geltungsbereich der Finitheits-Kategorie hat sich jedoch in der Folgezeit auf die Beschreibung von Verbformen ausgeweitet, mit dem Ergebnis, dass sie heute traditionell als Kategorie der verbalen Flexionsmorphologie eingeordnet wird. ${ }^{7}$ Finite Verb-

6 Vgl. Nikolaeva (2007:1): „The term ,finite“ adopted in European linguistics goes back to the Latin finitus, the perfective participle of the verb finio ,finish, limit, set bounds to, determine“" .

7 Der flexionsmorphologische Ansatz bei der Markierung von Finitheit hat hier allerdings keine universal-typologische Relevanz, da die Unterscheidung zwischen finiten und infiniten Formen auch in den flexionsmorphologisch schwach ausgeprägten Sprachen (wie etwa dem Englischen) getroffen wird. Die Fi- 
formen sind demnach solche, die hinsichtlich der Hauptkategorien des Verbs (Numerus, Person, Tempus, Modus) markiert sind. Typologisch gesehen können allerdings keine eindeutigen Aussagen darüber gemacht werden, welche (verbalen) Kategorien die Finitheit konstituieren. In vielen Fällen ist es nahezu unmöglich, eine trennscharfe Grenze zwischen finiten und nichtfiniten Formen zu ziehen. Beispielsweise können im Portugiesischen sogar anscheinende Infinitive mit finiten Kategorien wie etwa der Personenmarkierung vorkommen (vgl. JOSEPH 1983), so dass sie sich einer klaren Einordnung als genuin nichtfinite Formen weitgehend entziehen. Um das Problem der typologisch uneinheitlichen Kodierung von Finitheit zu umgehen und den jeweiligen Finitheits-Konzepten, die einzelsprachlich-relativ angelegt sind und der genuinen Spezifik der Einzelsprache Rechnung tragen, eine übereinzelsprachliche Relevanz zu verleihen, wurde als generalisierendes Kriterium das autonome Bestehen von finiten Formen angenommen (vgl. z. B. GIVóN 1990). Die Existenz von nichtfiniten, syntaktisch selbstständigen Sätzen läuft jedoch auch dieser Faustregel entgegen.

In der modernen Grammatikforschung hat sich eine Vielzahl von unterschiedlichen Schwerpunktsetzungen und Herangehensweisen bei der Behandlung der Finitheit als der verbalen Kategorie etabliert. Das Hauptinteresse der Finitheitsforschung konzentriert sich auf typologisch begründete Fragestellungen. Im Mittelpunkt der sprachwissenschaftlichen Diskussionen stehen dabei vor allem Probleme der einzelsprachlichen Finitheitsmarkierung oder unterschiedlich fundierte Versuche, die kategoriale Beschaffenheit von Finitheit in den jeweiligen Sprachsystemen zu definieren. Eine wichtige Problemstellung richtet sich hierbei auf die bereits oben erwähnte Frage, welche der grammatischen Kategorien des Verbs (Numerus, Person, Tempus, Modus etc.) die kategoriale Bedeutung von Finitheit konstituieren (vgl. EISENBERG 2006; CRISTOFARO 2007; NiKOLAEVA 2007).

In der deutschen Sprache wird Finitheit nicht nur als Kategorie der Verbalflexion behandelt, die sich in der formalen Variabilität des Verbs hinsichtlich seiner grammatischen Kategorien manifestiert. Verbale Finitheitsmarker spielen auch im Bereich der Syntax eine Rolle, da die verbale Prädikation im deutschen Satz prototypisch durch ein finites Verb kodiert wird. Das Vorhandensein einer finiten Verbform in einem Syntagma wird in den meisten

nitheitsmarkierung erfolgt hier nicht bzw. nicht nur mittels morphologischer Marker, sondern ist vielmehr an eine Reihe von syntaktischen, semantischen und/oder pragmatischen Kodierungsmitteln gebunden. 
Grammatiken zur deutschen Sprache, wie eingangs ausgeführt, als das wichtigste satzkonstituierende Kriterium behandelt (vgl. u. a. ZIFONUN 1997; ENGEL 1996). Folgt man diesen Satzkonzepten konsequent, sind verblose Zeichensysteme wie etwa Feuer!; Pech gehabt; Schade; alles klar! etc. grundsätzlich nicht als Sätze, sondern als satzäquivalente Äußerungen mit kommunikativem Wert oder - wie ZIFONUN (1997:86f.) vorschlägt - „,kommunikative Minimaleinheiten“ anzusehen. Das Kriterium der Finitheit als strukturell-semantisches Satzmerkmal darf jedoch - typologisch gesehen keine universelle Geltung haben und nicht als der absolute Satz-Indikator auf alle anderen Sprachen angewendet werden: Denn so richtig es ist, die overten Kodierungsformen im Bereich der verbalen Prädikationen als prototypische Satzmuster im Deutschen anzusetzen, so richtig ist es auch, z. B. verblose Konstruktionen mit dem deiktischen Kopulapronomen to (,das') im Polnischen (vgl. etwa Piotr to geniusz, Peter ist ein Genie“ [wörtl. Peter das Genie]) oder nominale Konstruktionen mit Nullkopula (vgl. etwa Kto tam? - To ja, Piotr! ,Wer ist da? - Ich bin es, Peter' [wörtl. Wer da? - Das ich, Peter]) als grammatisch korrekte, unmarkierte ,Sätze` zu behandeln. Auch bei Satzverhältnissen der früheren Entwicklungsstufen der germanischen Sprachen, in denen nicht-finite Satzmuster relativ häufig belegt sind, ja manchmal kumulativ vorkommen, muss der Anspruch auf Exklusivität der Verbzentriertheit als des übergeordneten satzkonstituierenden Kriteriums ipso facto relativiert werden. Im germanischen Sprachbereich betrifft dies insbesondere das Gotische, in dem größere Teile der Satzgebilde sehr oft kein finites Verb enthalten. Diese typologisch relevante Besonderheit verbindet das Gotische mit dem Griechischen. Die Ähnlichkeiten sind jedoch nicht linear, so dass die gotischen infiniten Satzkonstruktionen nicht unbedingt als isomorphe griechische Entsprechungen zu beurteilen sind (vgl. KoTIN 2012:321f.).

Die diachron-historischen Beobachtungen der sprachhistorischen Verhältnisse am deutschen Sprachmaterial legen nahe, dass den overten Finitheitsmustern im verbalen Bereich vielfach koverte Prädikationsmodelle zu Grunde lagen und dass der stufenweise Übergang von dem koverten zu dem overten Prädikationsmuster eine Reihe von Wandelprozessen in unterschiedlichen Ebenen der deutschen Sprache ausgelöst hat. Diese haben in der Synchronie nicht nur zu verschiedenen kategorialen oder grammatischen Abhängigkeiten (z.B. im Bereich der Konnektoren oder der Kasussyntax) geführt, sondern auch weitgehende Folgen für die syntaktisch-topologische Strukturierung von Sätzen mit sich gebracht. 


\section{Zum Begriff der ,Prädikation'}

In dieser Abhandlung wird von der Prädikation als dem wichtigsten satzkonstituierenden Kriterium ausgegangen. Prädikation gehört, neben Nomination, zu den wichtigsten sprachschöpferischen Tätigkeiten ${ }^{8}$ des sprechenden Menschen (vgl. KoTIN 2007). Von der Nomination unterscheidet sich der Prädikationsakt dadurch, dass er nicht einzelne Wortschatz-Entitäten ${ }^{9}$, d.h. , langue'-Einheiten kreiert, sondern hierarchische Dependenzrelationen zwischen den einzelnen, in einer Zeichenkette linear angeordneten und unterschiedlichen grammatischen Kategorien angehörenden sprachlichen Entitäten schafft. Mit der Formierung eines jeden Satzes geht also die Bildung dependenter Relationen einher. Insofern ist es unmöglich, einen prädikationslosen Satz zu bilden. ${ }^{10}$ Die Quelle für die Prädikationsrelationen und somit für die Satzbildung ist das verbale Prädikat, das auf der syntaktischen Ebene die Rolle des Regens übernimmt und unterschiedliche (nominale oder adverbiale) Dependentia anschließt. ${ }^{11}$ Das Wesen der verbalen Prädikate hat HERIN-

8 Insofern kann man sie im Sinne Humboldts als, energeia‘ bezeichnen.

$9 \quad$ Ich abstrahiere hier der Einfachheit halber von komplexen Nominationseinheiten wie etwa den phraseologischen bzw. idiomatischen oder im Zuge der Wortbildung entstandenen Nominationssyntagmen.

10 Von dieser Regel sind Modalitätssätze mit epistemischer Funktion oder - allgemeiner - übergeordnete Satzoperatoren ausgenommen, da sie bekanntlich keine Prädikationen, sondern sprecherbezogene Einstellungen zu den jeweiligen Prädikationen enthalten.

11 Es wird hier dependenzgrammatisch vorgegangen, wobei das Verb die dominierende Rolle bei der konstruktionellen und semantischen Satzbildung spielt. Das syntaktische Dependenzkonzept ist nicht das einzige Syntaxmodell, das für die Analyse von Sätzen als sprachliche Kodierungsmuster für prädikative Relationen herausgearbeitet wird. Es gibt auch Konzepte, bei denen nicht das Verb, sondern gerade das Subjekt im Zentrum des Interesses steht und für die Herstellung von prädikativen Relationen zuständig ist. So gesehen, bildet das Subjekt als syntaktische Objektivierungsform für einen (in ontologischen Kategorien ausgedrückten) Gegenstandsbegriff den zuerst im Bewusstsein des sprechenden Menschen aufkommenden Vorstellungsinhalt und steht demzufolge am Anfang eines jeden sprachlichen Denkprozesses. Dadurch eröffnet es auf ganz natürliche Weise eine Erwartungsperspektive, durch den Bestimmungsbegriff (das Prädikat) konkretisiert zu werden (mehr dazu vgl. u.a. vON DER GABELENTZ 1868; PAUl 1995; DONHAUSER 2007). In diesem Artikel wird die dominierende Funktion bei der Satzbildung allerdings dem Verb als der ,Quelle' der Prädikation zugesprochen. 
GER (1984:49) sehr treffend identifiziert, der die Prädikate als szenenbildende Sprachinstanzen auffasst: „Das Verb, das ist so, wie wenn man in einem dunklen Raum das Licht anknipst. Mit einem Schlag ist die Szene da." Die Prädikation wird somit durch die Gesamtheit aller innerhalb einer Szene bestehenden Relationen zwischen den jeweiligen Mitspielern konstituiert. Sie ist ein kognitives Muster für prototypische Abläufe von Ereignissen, Handlungen und Zuständen, an denen Subjekte und Objekte unterschiedlicher Art teilnehmen. Beispielsweise werden in die durch das verbale Prädikat kaufen kreierte Kaufszene die Rollen von einem Käufer und einem Kaufobjekt eingebunden; die Kaufszene setzt aber auch die Existenz eines Verkäufers voraus, sie spielt sich an einem bestimmten Verkaufsort, zu einen bestimmten Zeitpunkt ab (vgl. KÖLLER 2004:388).

Die Bildung von Prädikationsrelationen beginnt auf der relationslogischen Ebene des Satzes, wo zwischen den Satzgliedern als den Repräsentanten unterschiedlicher Rollen unterschiedliche Abhängigkeitsrelationen hergestellt werden. Insofern vereint der Satz zweierlei Funktionen in sich: Er ist eine in sich geschlossene, selbstständige sprachliche Äußerungseinheit, zugleich aber ein Spiegelbild der durch das Prädikat als die zentrale Ordnungsinstanz des Satzes kreierten Relationen zwischen den jeweiligen (einer Szene angehörenden) Satzentitäten. Abgesehen von Sprachen wie z. B. dem Chinesischen, wo es bekanntlich keine klar ausgeprägten Wortarten und dementsprechend auch keine Satzglieder im herkömmlichen Sinn gibt und wo auf Grund dessen die Zuordnung von Prädikationsrelationen womöglich anders verläuft, lassen sich Prädikationsrelationen als logische Ordnungsstrukturen auffassen, die jeder Satzbildung in jeder Sprache zu Grunde liegen. Ihre Projektion auf die terminative Satzstruktur muss jedoch nicht in expliziter Form, d.h. mittels des finiten Verbs erfolgen, sondern kann kovert kodiert werden. Deshalb muss zwischen Prädikation und dem Vorhandensein einer finiten Verbform (Finitheit) kein direkter Zusammenhang bestehen. Die koverten Prädikationen lassen sich auf eine mehr oder weniger umständliche Weise in overte Prädikationen überführen. Koverte Prädikationen werden zunächst rein intuitiv rekonstruiert. Hinter den intuitiven Rekonstruktionsmechanismen stehen aber grammatische Wissensbestände, durch die unser Denken und Sprechen strukturiert werden. Auf inhaltlich-semantischer Ebene ist es vor allem die Zuordnung koverter Prädikationen zu einem der semantischen Prototypen wie etwa Handlung, Ereignis, Zustand, Existenz, Vorhandensein, Besitz etc., wofür es in der Regel prototypische Verben gibt, also entsprechend tun, geschehen, sein, es gibt, haben (vgl. KOTIN 2007:197). 


\section{Koverte Finitheitsmuster}

\subsection{Koverte Finitheitsmuster im Gotischen}

Im Folgenden wende ich mich konkreten historischen und synchronischen Textbelegen zu, um das Phänomen der koverten Finitheit bzw. Prädikation ${ }^{12}$ näher zu bringen. Ich fange mit der Analyse der gotischen Sätze an. Infinit kodierte Satzprädikationen sind geradezu das charakteristische Merkmal der gotischen Syntax (vgl. KotiN 2012:321), wobei sie in die Struktur eines einfachen Satzes als nomendependente Ergänzungen eingebettet werden. Das bedeutet, dass ein Satz mit einem Verbum finitum als dem zentralen, auf der Ausdrucksseite realisierten Prädikat mehrere verbale Prädikationen in sich enthalten kann, die in den meisten Fällen nicht durch eine finite Verbform, sondern die mit den substantivischen Satzgliedern bzw. Satzgliedteilen kongruierenden Partizipialattribute kodiert werden. Auf diese Weise entsteht ein prädikativ komplexes Gebilde, ja eine verbale „Portmanteau-Prädikation“, in der mehrere verbale Prädikationen zugleich zum Ausdruck kommen, vgl. hierfür das folgende Beispiel aus der gotischen Wulfila-Bibel:

(1) (Mt. 8, 2) jah sai, manna prutsfill habands durinnands inwait ina qipands: frauja, jabai wileis, magt mik gahrainjan.

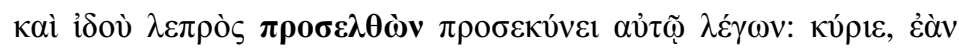

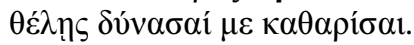

[wörtlich]: ,und siehe, ein Mann Aussatz habender zurennender grüßte ihn sprechender: Herr, wenn (du) willst, kannst (du) mich rein machen.

Die wortwörtliche Übersetzung des gotischen Satzes ist derart kompliziert, dass sie nahezu an der Grenze der Verständlichkeit steht. Dennoch zeigt sie eindrucksvoll, wie eine verbale Prädikation durch infinite, und zwar partizipiale Formen kodiert werden kann. Das erste Partizip habands, um das es sich hier handelt, ist gemäß seinem syntaktischen Status durch das nominale Regens manna gebunden und wird attributiv verwendet. Auf der anderen Seite verhält es sich wie ein typisches transitives Verb, das valenzgramma-

12 Die Begriffe ,koverte Finitheit' und ,koverte Prädikation ‘ werden im vorliegenden Beitrag trotz ihrer referentiellen Affinität nicht synonymisch verwendet, denn sie stellen jeweils einen anderen Aspekt des hier behandelten Problemkomplexes in den Vordergrund: Mit koverter Finitheit wird lediglich darauf verwiesen, dass an der terminativen Ebene des Satzes kein finites Verb auftritt. Die koverte Prädikation bezieht sich eher auf das Ergebnis der Aussparung der finiten Verbformen. 
tisch gesehen einer Ergänzung im Akkusativ (brutsfill) bedarf. Eine mit ihm vergleichbare janusköpfige Form ist das nachfolgende Partizip durinnands, das ebenfalls als Dependens dem Kopf der Nominalphrase manna beigeordnet wird, in seiner Semantik aber eine verbale Handlung ausdrückt und somit als ein klassisches Prädikat zu behandeln ist. Auch das dritte Partizip qipands wird durch das nominale Regens manna etabliert und fungiert als sein Komplement, dabei wiederum eine verbale Prädikation kodierend (vgl. KotiN 2012:321f.). Wir haben hier also ein interessantes Paradoxon vor uns, dass nämlich - formal gesehen - alle drei partizipialen Formen von dem Nomen gebunden werden; valenzgrammatisch liegt hier jedoch eine umgekehrte Situation vor: Es ist das Nomen, das von den Partizipien gebunden wird, und zwar als ihre Nominativergänzung. Somit ergibt sich, dass der behandelte Satz insgesamt über vier Marker verfügt, die die verbale Prädikation zu etablieren vermögen, wobei nur einer davon die prototypische Form eines overten finiten Verbs hat. Die übrigen drei Prädikationsmarker kommen als partizipiale, und somit koverte Mittel vor. In dem behandelten Satz (1) sieht die prädikative Struktur folgendermaßen aus: Ein Mann, der Aussatz hatte (die 1. (koverte) Prädikation), lief auf ihn zu (die 2. (koverte) Prädikation), grüßte ihn (die 3. (overte) Prädikation) und sprach [...] (die 4. (koverte) Prädikation). Alle Prädikate teilen sich also dasselbe Subjekt. Schematisch lässt sich die prädikative Struktur des Satzes wie folgt darstellen:

overte Prädikation (3)

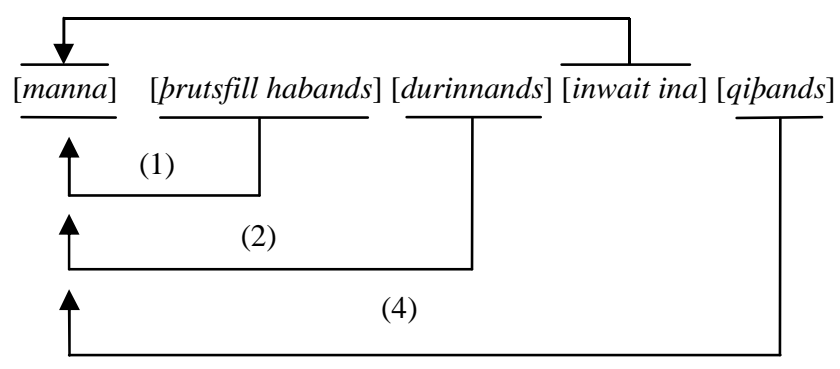

koverte/infinite Prädikation 
Der Parallelsatz aus dem Lukas-Evangelium passt sich hinsichtlich seiner Prädikationsstruktur in dasselbe Schema ein:

(2) (Lk. 5,12): mippanei was is in ainai baurge [...] manna fulls prutsfillis jah gasaihvands Iesu driusands ana andwairpi bad ina qibands: frauja, jabai wileis, magt mik gahrainjan.

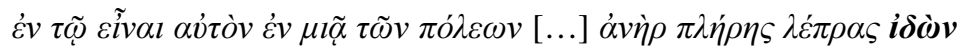

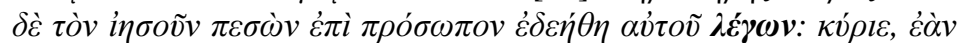
$\theta \dot{\lambda} \lambda \eta \varphi_{\varsigma} \delta \dot{v} v \alpha \sigma \alpha i \dot{\mu} \mu \varepsilon \alpha \theta \alpha \rho i \sigma \alpha l$.

[wörtlich]: ,Während er [Jesus] in einer der Städte war ein Mann voll von Aussatz und erblickender Jesus niederfallender auf sein Gesicht bat ihn sprechender: Herr, Wenn (du) willst, kannst (du) mich rein machen.

Der dritte Parallelbeleg, der die Geschichte der Heilung eines Aussätzigen behandelt, kommt aus dem Markus-Evangelium. Im Gegensatz zu den zwei oben aufgeführten Satzbelegen, liegt hier eine Besonderheit bei der Einbettung der koverten Prädikate vor, nämlich die, dass die koverten Prädikate nicht von Substantiven etabliert, sondern direkt von dem finiten Verb gebunden werden:

(3) (Mk. 1, 40): jah qam at imma prutsfill habands, bidjands ina jah kniwam knussjands jah qipands du imma patei jabai wileis, magt mik gahrainjan.

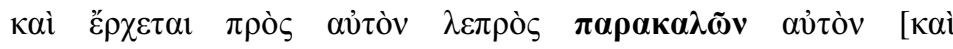

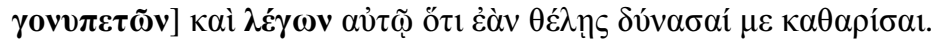

[wörtlich]: ,Auch kam zu ihm Aussatz habender, betender ihn und kniender und sprechender zu ihm: Wenn (du) willst, kannst (du) mich rein machen.

Das aktive Partizip habands (,habender') wird vom finiten Verb qam (,kam') direkt etabliert. Genauso wie im Beleg 1 realisiert das Partizip als Ableitung des transitiven Verbs auch hier seine Objektvalenz (prutsfill). Das Besondere besteht aber darin, dass dieses Partizip als koverter Prädikatsmarker die Funktion des Satzsubjekts übernimmt: Es steht nämlich metonymisch für das nicht genannte Agens. Vergleichbares gilt auch für die weiteren Partizipien bidjands, knussjands, qibands, wobei das letztere Partizip seine Objektvalenz in Form einer satzförmigen direkten Rede realisiert. Alle drei Partizipien fungieren in formal-grammatischer Sicht als Satzsubjekte, syntaktischsemantisch kodieren sie aber verbale Prädikationen. Anzumerken ist ferner, dass zwischen dem griechischen Vorlagetext und der gotischen Übersetzung keine durchgehenden Entsprechungen bei der Kodierung von koverten Prädi- 
kationen vorliegen. Alle drei Beispiele zeigen, dass das Gotische die griechischen Satzmuster zwar nachbildet, aber eben nicht konsequent und nicht textübergreifend. Hingewiesen sei lediglich auf den dritten Beleg, in dessen griechischem Textteil anstelle der Partizipialform das einfache Substantiv $\lambda \dot{z} \pi \rho \alpha \varsigma$ (,Aussätziger') steht. Somit kann nicht pauschal davon ausgegangen werden, dass die koverten Finitheitsmuster im Gotischen ein im Zuge der mechanischen Lehnübersetzung griechischer Originalstrukturen entstandenes Syntaxphänomen ist. Vielmehr handelt es sich um ein altererbtes gemeinindogermanisches Phänomen, dessen Kodierungsmittel in vielen Sprachsystemen, darunter im Gotischen, als syntaktischer Archaismus konserviert worden sind.

Bei koverter Kodierung verbaler Prädikationen liegt eine Unterspezifizierung der Prädikate hinsichtlich der verbalen Kategorien wie etwa des Tempus oder des Modus vor. Mit anderen Worten bedeutet dies, dass die infinit kodierten Prädikationen weder eine explizite zeitliche Perspektivierung der Sachverhalte noch eine Markierung ihrer Faktizität aufweisen, wie sie sonst in den finiten Satzmustern durch das Verbalmorphem des jeweiligen finiten Verbs gekennzeichnet werden. Sie sind vielmehr in der Hauptprädikation verankert und übernehmen alle verbalkategorialen Bedeutungen des finit realisierten Hauptprädikats. Die finite Verbform des Hauptprädikats liefert demnach wichtige Spezifizierungen der Verbalsemantik wie etwa Temporalität, Modalität und/oder Faktizität. Insofern bildet die finite Hauptprädikation, die dem ganzen Satz zu Grunde liegt, den prädikativen Bezugsrahmen für alle infinit kodierten Teil-Prädikationen. Das prädikative Gerüst der gesamten Verbalhandlung bildet in den analysierten Textbeispielen zweifelsohne die overte Prädikation (in Beleg 1 manna inwait ina , ein Mann grüßte ihn'; in Beleg 2 manna fulls prutsfillis bad ina, ein Mann voll von Aussatz bat ihn“; Beleg 3 qam prutsfill habands, ein Aussätziger kam‘). Sie determiniert den Zeitpunkt (Vergangenheit) der jeweiligen verbalisierten Ereignisse, die in der zeitlichen Sukzession nacheinander verlaufen, was auch ikonisch durch die topologische Anordnung der koverten Prädikate wiedergegeben wird. ${ }^{13}$

13 Es ist üblich, dass wir jede erzählte Geschichte im Sinne der nacheinander folgenden verbalen Handlungen bzw. Ereignisse verstehen. Die verbalen Prädikationen verlaufen jedoch nicht immer zeitlinear. Manchmal werden die dadurch kodierten Handlungen als nachzeitig dargestellt, andere werden vorweggenommen. All dies wird typischerweise mit Hilfe von Satzkonnektoren zum Ausdruck gebracht. Im Fall der kovert kodierten Prädikationen haben wir es nicht mit den die einzelnen Sätze verknüpfenden Konnektoren zu tun, sondern vielmehr mit 


\subsection{Koverte Finitheitsmuster im Althochdeutschen}

Ein interessantes Zusammenspiel von kovert und overt kodierten Prädikationen findet sich auch im nachfolgenden Satz aus dem althochdeutschen Tatian-Text:

(4) (Tat. 119, 28-120, 7): antvvurtenti thó p\&rus quad/ trohtin ob thúz bist heiz mih queman /zi thir ubar thisiu uиazzar/ thara uuidar her thó quad quim/Inti nidarstiganter p\&rus/fon themo skefe gieng oba/ theто uиazare [...] Inti so her bigonda sinkan/riof quedanter. truhtin, heilan/tuo mih

Respondens autem $p \&$ rus dixit./domine si tu es. iube me uenire/ad te super aquas/at ipse ait. ueni. I\& descendens $p \&$ rus de navicula ambulabat super aquam [...] \& cum coepiss \& mergi/clamauit dicens. Domine. saluum/me fac

[wörtlich]: ,Antwortend da Petrus sprach: Herr, wenn du es bist, lass mich über das Wasser zu dir kommen. Darauf sprach er: Komm! Und steigender Petrus aus dem Schiff ging auf dem Wasser [...] und als er zu sinken begann, rief sprechender: Herr, rette mich!'

Wiederum haben wir es hier mit partizipialer Kodierung der Prädikationen zu tun. Die partizipialen Prädikate werden hier, ähnlich wie in den Beispielen zuvor, durch ein Regens gebunden, wobei im ersten Satz ein unflektiertes Partizip I (antvvurtenti) vorliegt, in zwei weiteren Fällen flektierte Partizipialformen (nidarstiganter, quedanter) auftreten. Das unflektierte Partizip antvvurtenti weist hier eine Doppelbindung auf: Es ist zum einen durch das nominale Regens (Petrus) etabliert, zum zweiten durch das overte Finitum (quad) selbst. Somit ist es nicht nur verbal geprägt (Petrus antwortete), sondern zugleich auch adverbial beschaffen (Petrus sprach, indem er antwortete). Die flektierten Partizipialformen treten als verbale Merkmalsträger in der dem Adjektiv ähnlichen Merkmalsfunktion auf. Sie fungieren, so gesehen, als Komplemente des nominalen Regens, weisen jedoch eine stark verbale Ausprägung auf. Insbesondere betrifft dies das Partizip des Fortbewegungsverbs nidarstigan (,(aus)steigen'), das hier durch die vollständige Realisierung seines Valenzprogramms (Nominativergänzung, Adverbialergänzung) an den

satzübergreifenden Textkonnektoren, die den Text als Ganzes steuern. Dabei kommen die semantischen (temporalen, kausalen etc.) Relationen zwischen den jeweiligen (Text-)Sätzen und den dadurch kodierten Prädikationen oft nicht deutlich genug zur Geltung. 
Ausdruck einer verbalen Prädikation besonders stark gebunden ist und sich demzufolge als klassischer verbaler Valenzträger verhält. Derartige Konstruktionen sind im heutigen Deutsch zwar denkbar, syntaktisch aber eher problematisch, denn die Satzbildung mittels koverter Finitheit führt zur Entstehung prädikativ, überstrapazierter' Sätze, in denen die jeweiligen Prädikationen nicht immer linear aufeinander folgen, sondern vielfach semantisch ineinandergreifen. Eine direkte Folge davon ist, dass die Bildung von Paraund Hypotaxen zum Teil blockiert wird. ${ }^{14}$ Darüber hinaus ist die Rezeption des Satzes, in dem die Prädikationsstruktur keine lineare Ordnungsstruktur aufweist, erheblich erschwert. Die Bildung von neuhochdeutschen Sätzen erfolgt daher prototypisch nach dem Grundsatz: Jeder Prädikation entspricht auf der Ausdrucksseite je ein finites Verb. So gesehen, müsste die neuhochdeutsche Übersetzung des behandelten althochdeutschen Satzes um drei finite Verbformen erweitert werden: ,Da antwortete (1) Petrus und sprach: Herr, wenn du es bist, lass mich über das Wasser zu dir kommen. Darauf sprach er: Komm! Und als er aus dem Schiff stieg (2), ging er auf dem Wasser [...] und als er zu sinken begann, rief er und sprach (3): Herr, rette mich! ‘

Eine direkte Folge der Satzbildung mittels koverter Verbalprädikationen ist die Tendenz zur Verwendung von absoluten Kasuskonstruktionen. Im Althochdeutschen (wie im Gotischen) gehört dazu vor allem die Bildung der Partizipialkonstruktionen im Dativ, die oft in Nachbildung des lateinischen Ablativus absolutus oder (im Fall des Gotischen) der griechischen absoluten Genitiv-Konstruktionen als syntaktisch autonome Konstruktionen auftreten und eine temporal bzw. eine temporal-konditional zu interpretierende Prädikation kodieren:

(5) (Tat. 81, 18-20): thó ziganganemo themo uúine/quad thes heilantes muoter zi imo./sie nihabent uúin.

\& deficiente uino./dicit mater ihesu ad eum.,/uinum non habent.,

[wörtlich]: ,Dann zu Ende gegangenem dem Wein [=als es an Wein mangelte], sprach die Mutter des Heilands zu ihm: Sie haben keinen Wein mehr.

(6) (Tat. 86, 11-12): Imo stigantemo in skef/folg\&un imo sine iungiron Et ascendente eo in nauicula/secuti sunt eum discipuli eius ${ }^{15}$;

14 Eine solche Folge ist tatsächlich im Gotischen eingetreten, mehr dazu vgl. KoTIN (2012:324f.).

15 Der gotische Parallelbeleg (Mt. 8, 23: jah innatgaggandin [Part. I Dat. Sg.] imma in skip, afariddjedun imma siponjos is) enthält ebenfalls einen Dativus ab- 
[wörtlich]: ,Ihm, dem Steigenden in das Schiff [=als er in das Schiff stieg], folgten ihm seine Jünger.

\subsection{Koverte Finitheitsmuster im modernen Deutsch}

Aus der Gegenüberstellung von den hier angeführten, aber auch in anderen Kontexten vorkommenden althochdeutschen und neuhochdeutschen Sätzen ergibt sich u.a., dass koverte Finitheitsmuster ein typisches Merkmal der historischen Sprachen sind, während sich die modernen Sprachsysteme gegenüber Infinitheit eher resistent verhalten. Das bedeutet aber nicht, dass uns koverte Prädikationsmuster heute überhaupt nicht begegnen. Sie leben nämlich fort, auch wenn ihr Vorkommen meist mit stilistisch-emphatischer Satzmarkierung einhergeht. Im modernen Deutsch lässt sich eine Reihe von unterschiedlich strukturierten kovert kodierten Prädikationsmustern differenzieren:

(7) Feuer!

(8) Er-und Geschirr spülen?

(9) Nie im Leben!

(10) Zurück zum Thema!

(11) Du auch?

(12) Wer? Ich?

(13) Wohin fahren?

(14) Was für eine Frechheit!

(15) In Ordnung!

(16) Auf dem Tisch.

(17) Bitte wenden.

(18) Rauchen nicht gestattet.

(19) Mutmaßlicher Täter festgenommen.

(20) Keine Ahnung.

(21) Er ging ins Kino und Maria ins Theater.

Die jeweiligen koverten Prädikationen sind, was ihre Kombinierbarkeit z.B. mit Temporalmarkern anbelangt, relativ beschränkt. Die Prädikationen, die mittels der subjektlosen Infinitivsätze kodiert werden, können in der tempora-

solutus, der die im griechischen Text vorkommende absolute Dativkonstruktion

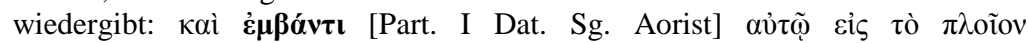

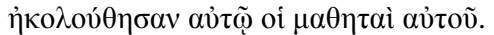


len Perspektive der Vergangenheit wohl kaum verwendet werden, wohingegen eine futurische bzw. eine futurisch-prognostische Interpretation eher unproblematisch ist. Die Distribution der koverten Prädikationsmuster beschränkt sich jedoch nicht nur auf einzelne Sätze, die atomar in einem Text vorkommen. Es lassen sich auch Belege finden, in denen relativ umfassende Text-Sequenzen als koverte Prädikationen gebildet werden, so auch im folgenden Beleg, in dem es bis auf den einleitenden Fragesatz, der die overte ist-Prädikation enthält, durchgehend koverte Prädikationsmuster gibt:

(22) Was ist deutsch? Tannenbäume? Reiseträume? Kühler Verstand? Kaltes Herz? Tiefsinn? Ausländerhass? Offenheit? Betroffenheit? Baseballschläger? [...] Sich immer schuldig fühlen? Sich Mut ansaufen? Den Verstand unterlaufen? [...] Die Selbstzweifel mit Schnaps wegspülen? Sich danach etwas besser fühlen? Pflichtbewusstsein? Ich? Ohne Fleiß kein Preis? Reisen ins Ausland? Ein Ferienhaus in Spanien? Das Auto aus Japan ${ }^{16}$

Davon zu unterscheiden sind Konstruktionen vom Typ der rote Wein, bei denen keine Prädikation, sondern eine Attribution vorliegt. Beide Begriffe müssen einander nicht streng dichotomisch gegenübergestellt werden, denn sowohl hier wie dort liegen vergleichbare Operationen vor ${ }^{17}$ : Ähnlich wie bei der Prädikation werden auch bei der Attribution relationale Determinationsbzw. Abhängigkeitsverhältnisse zwischen dem Regens (als dem Kopf der Nominalphrase) und dem dazu gehörigen (attributiven) Dependens bewerkstelligt, die sich etwa als Zuweisung einer Eigenschaft (in dem konkreten Fall: die Farbe rot) an eine Entität (in dem konkreten Fall: Wein) identifizieren lassen, wobei die Dependenz nicht vom Attributiv, sondern vom regensfähigen Nomen ausgeht. EISENBERG (1986:215) ordnet daher die gesamte Konstruktion zu Recht der Kategorie Nominalgruppe zu. Auch aus sprachgenealogischer Sicht können Attribution und Prädikation als eng verwandte operationelle Verfahren angesehen werden: Es wird angenommen, dass es in den frühesten Etappen der Sprachenentwicklung keine so deutliche Trennung zwischen Verb und Substantiv, ja zwischen Substantiv und Adjektiv gab, wie wir sie heute kennen (vgl. KOTIN 2012:323), so dass zwischen den satzinternen (verbozentrischen) und syntagmainternen (nominalzentrierten) Hierarchien keine scharfen Unterschiede bestanden. Es steht jedoch außer Zweifel,

16 http://egora.uni-muenster.de/FmG/fremdenfeindlichkeit/m1207.shtml (15.01.14).

17 Die Attribution innerhalb einer Nominalgruppe wird daher als sekundäre/abgeleitete Prädikation (Der Wein ist rot $\rightarrow$ der rote Wein) angesehen (vgl. TEUBERT 1979; HelBig / BusCHA 1987). 
dass verbale und „nominale“ Regensfunktionen jeweils etwas Anderes leisten und auf anderen Hierarchie-Ebenen beheimatet sind. Die verbalen Dependenzrelationen verlaufen in einer der attributiven Dependenz übergeordneten Ebene und kreieren auf Grund dessen Prädikationen, die ihrerseits die Quelle der Satzbildung sind. Demgegenüber ist die Attribution in einer tieferen Ebene angesiedelt. Attribute sind Komplemente von Satzgliedern (Subjekten), die selbst als Komplemente (des Verbs) fungieren und zusammen mit dem „,nominalen Regens“ das Teilsyntagma bilden, das auf der (höher gelegenen) Prädikationsebene als subjektales Dependens auftritt. ${ }^{18}$

\subsection{Koverte Finitheitsmuster im Polnischen}

In den slawischen Sprachen, wie etwa dem Polnischen, ist die partizipiale Finitheitsmodellierung ein mit den altgermanischen Sprachen vergleichbarer, wenngleich keineswegs deckungsgleicher Mechanismus der koverten Prädikationskodierung. Die partizipialen Satzmuster stellen im Polnischen dabei nur eine der vielen Techniken ${ }^{19}$ der impliziten Prädikationskodierung dar. Vergleicht man die oben behandelten historischen Sätze mit den Parallelbelegen aus der polnischen Bibelübersetzung, so lässt sich feststellen, dass die polnischen Satzstrukturen ganz stark den diachronen Satzmustern ähneln:

18 Aus diesem Grunde werden Attribute in den herkömmlichen, dependenzorientierten Grammatiken (vgl. z. B. ÁGEL / EICHINGER / EROMS 2003:1014) nicht als Satzglieder, sondern als Satzgliedteile behandelt.

19 Eine der interessanten Techniken der koverten Prädikationskodierung sind auch die sogenannten Nominalsätze. Ein Nominalsatz ist ein Satzgebilde, das aus isolierten Substantiven im Nominativ ohne oder mit untergeordneten Satzgliedern besteht, vgl. etwa Cisza (,Stille'), Deszcz (,Regen'), Jutro wyjazd (,Morgen Abfahrt'). Im Nominalsatz sind keine syntaktischen Positionen unbesetzt, weswegen er nicht als elliptische Konstruktion zu interpretieren ist. Der Satz eröffnet nur eine einzige syntaktische Position, die durch das Substantiv im Nominativ besetzt wird. Die Nominalsätze können jedoch durch direkte Ergänzung zu einer Konstruktion mit overter Prädikation übergehen: Jest cisza (,Es ist/herrscht Stille'), Pada deszcz (,Es regnet'), Jutro będzie wyjazd (,Morgen ist Abfahrt'). Die Nominalsätze sind vor allem in der Kinder- und Umgangssprache stark verbreitet. Sie werden auch in Überschriften, Titeln oder Aufschriften aller Art verwendet. 
Monika Schönherr

(23) (Mt 14, 29-31):

[Piotr]

Piotr-NOM.SG.

widząc

gwattowna

wichurę,

sehend-PART.

starken-AKK.SG

Wind-AKK.SG.

przestraszyt się $i \quad$ zacząt tonać.

[...] A Jezus

fürchtete sich

und begann zu sinken

Und Jesus sogleich ausstreckend-PART. Hand-AKK.SG.

pochwycit go.

ergriff ihn-AKK.SG.

,Als Petrus den starken Wind sah, fürchtete er sich und begann zu

sinken. Sogleich aber streckte Jesus die Hand aus und ergriff ihn.

Ähnlich wie in den gotischen und althochdeutschen Belegen haben wir es hier mit einem Zusammenspiel von overt und kovert kodierten Satzprädikationen zu tun. Insgesamt wird der kurze Text aus fünf Prädikationen konstituiert, wobei zwei davon (widzac ,sehend', wyciagnawszy ,ausstreckend') in impliziter (partizipialer) Form vorliegen. An dem Beleg sieht man deutlich, dass sich die durch ein kovertes Mittel etablierte Prädikation und die overte Prädikation an der syntaktischen Oberfläche das gemeinsame Satzsubjekt teilen. Im Unterschied zu den altgermanischen aktiven Partizipien (Partizip I), die stets eine Gleichzeitigkeit der Handlung kodieren, lässt sich bei den polnischen partizipialen Prädikationsmarkern eine temporale Distinktion beobachten: Die Partizipien können - ähnlich den overten finit kodierten Prädikaten - entweder eine Vorzeitigkeit (wyciagnawszy) oder eine Gleichzeitigkeit (widzac) des verbalen Sachverhalts zum Ausdruck bringen. Es drängt sich die Frage auf, ob es sich bei den Adverbialpartizipien der Gleichzeitigkeit (widzac) und den Adverbialpartizipien der Nicht-Gleichzeitigkeit ${ }^{20}$ (wyciagnawszy) um Modalangaben handelt, welche den Vollzug des verbalen Vorgangs als dessen inhärente Eigenschaft spezifizieren bzw. modalisieren. Das ist nicht der Fall. Das Polnische kennt keine Modalsätze (vgl. SCHATTE 2011:242). Die Adverbialpartizipien dienen hier eindeutig nicht zur modalen Spezifizierung der Satzproposition, sondern sind selbst Prädikationen, auch wenn diese an der syntaktischen Oberfläche versteckt bleiben.

Interessant gestaltet sich hier aus kontrastiver Sicht zudem die Frage, welche Verben im Polnischen typischerweise als koverte Prädikate erscheinen. Eines der interessantesten Verben, dessen Verwendung bzw. Weglassung beson-

20 Beide Termini gehen auf GRZEGORCZYKOWA (2004:82) zurück. 
ders aussagekräftig ist, ist das Verb sein. ${ }^{21}$ Auf Grund der Umdeutung seiner archetypischen Semantik (Existenz) kann das sein-Verb in unterschiedlichen Kontexten vorkommen, dabei recht unterschiedliche semantische Relationen wie etwa Zustand, Lokativität, Possessivität, Klassenzugehörigkeit u.v.m. ausdrücken. Vergleichen wir nun folgende polnische Beispiele:
(24a) Piotr Peter-NOM.SG.
to
das
geniusz.
,Peter ist ein Genie.
(24b) Piotr byt Genie-NOM.SG. Peter-NOM.SG. war-3P.SG.PRÄT.MASK. Genie-INSTR.SG. ,Peter war ein Genie.

Es sind Satzmuster, in denen die Infinitheit nur an bestimmte kategoriale Bedeutungen gebunden ist. Es fällt auf, dass das Verbum substantivum lediglich im Präsens, also im Beispiel (24a) ausgelassen wird ${ }^{22}$. In der Vergangenheitsform ist diese Auslassung nicht mehr typisch und resultiert in den meisten Fällen in einer sloganartigen Äußerung mit einem elliptischen Charakter, vgl. Piotr geniuszem [Piotr-NOM. Genie-INSTR.]). Obendrein fällt auf, dass die infinite (a)-Äußerung eine obligatorische pronominale Konstituente to (,das') enthält. Die to-Konstituente ist kein anaphorisches Pronomen; sie kongruiert nicht mit dem Satzsubjekt und bleibt stets unflektiert. ${ }^{23}$ Vielmehr ist sie ein operatives Deiktikum. Die deiktischen Pronomina unterscheiden sich von den anaphorischen Pronomina u.a. dadurch, dass sie kein overt realisiertes Antezedens benötigen. Die Sätze mit der to-Konstituente können also auch ohne Antezedens auftreten, dieses muss aber für die Interpretation auf Grund des Vorkontextes (Kim jest Piotr ( $z$ zawodu)? - $\emptyset_{\text {Piotr }}$ to naukowiec , Was ist Piotr (von Beruf)? - das Wissenschaftler') oder auf Grund der Kontiguitätsrelationen (Na horyzoncie ukazat się pierwszy samochód wyścigowy - to Piotr ,Am Horizont erschien der erste Rennwagen - das Peter ${ }^{6}$ ) stets rekonstruierbar ist. Die operative Funktion des to-Elements besteht also

21 Näheres zum Verb sein vgl. KotiN (2013): „Sein. Eine genealogisch-typologische Fallstudie über das Verbum substantivum“. (Manuskript)

In derartigen Konstruktionen spezifiziert das to-Element das Satztempus als Präsens, den Satzaspekt als imperfektiv, den Satzmodus als Indikativ und das Genus Verbi als Aktiv.

23 Dies lässt sich z.B. an Linksherausstellungskonstruktionen veranschaulichen, vgl. etwa: Nasi przyjaciele - oni/*to zawsze stuża nam pomoca. (,Unsere Freunde, sie/*das stehen uns immer bei $\left.{ }^{`}\right)$. 
darin, dass es einer (meist in Form eines Frage- oder Relativsatzes) rekonstruierten Proposition mit einer Variablen (Wer den Rennwagen gefahren hat, ist Piotr) eine neue Proposition bzw. einen Wert für diese Variable (z. B. wie hier - ein Individuum bzw. ein Agnes) zuordnet. Das Deiktikum hat hier eine äquative Bedeutung, d.h. es kodiert eine koverte Identität/GleichheitPrädikation. Insofern ähneln die polnischen to-Sätze den englischen PseudoCleft-Sätzen (vgl. GEIST / BŁASZCZAK 2000:127), bei denen der erste Teil als freier Relativsatz erscheint und eine Fragesemantik aufweist und der zweite Teil eine elliptische Antwort auf diese Frage darstellt:

$$
\begin{array}{cc}
\text { a. } \quad \text { What Piotr } & \text { likes is his car } \\
\text { <-- Frage --> } & <-- \text { Antwort --> }
\end{array}
$$

b. What Piotr likes? - [Piotr likes his car]

c. What Piotr likes is [Piotr likes his car]

Informationsstrukturell gesehen steht die to-Konstituente - ähnlich dem finitem Verb - an der Grenze zwischen topikalen (vorerwähnten, bekannten) und fokalen (neuen, unbekannten) Satzelementen: Das von der to-Konstituente rechtsplatzierten Satzentitäten haben stets den größten Mitteilungswert und enthalten somit den Fokus des Satzes.

\section{Rückblick und Ausblick}

Die Existenz und die reguläre Verwendung von unterschiedlichen koverten und overten Finitheitsmustern veranlasst zu dem Schluss, dass man im Bereich der Kodierung von verbalen Prädikationen eigentlich nur schwer von einem bestimmten Routinemechanismus sprechen kann. Vielmehr existieren hier zahlreiche Kodierungstechniken parallel zueinander. Die unterschiedlichen Entwicklungswege der finiten und infiniten Satzmuster sind keineswegs als arbiträr oder ausschließlich sprachkontaktbedingt zu betrachten. Die Unterschiede in der Finitheitsausprägung und -markierung sind eher durch eine Reihe von sprachlichen (syntaktischen, semantischen oder pragmatischen) Faktoren motiviert. Die koverte Finitheit ist nicht einfach als ein der overten Finitheit (im Sinne einer klassischen privativen Opposition) gegenübergestelltes, merkmalloses Pendant zu beurteilen. Vielmehr handelt es sich - so das Fazit der Überlegungen - um ein mehr oder weniger eigenständiges Syntaxphänomen, das auf einem bestimmten Entwicklungsweg entstanden ist und mit finiten Satzmustern mannigfaltige Relationen eingeht. Finitheit ist ein skalares Phänomen, was bedeutet, dass in einem Satz ein Mehr an Finitheit, in einem anderen dagegen ein Weniger an Finitheit vorliegen kann. 
Überall dort, wo Finitheit nicht an der terminativen Oberfläche des Satzes durchschlägt, kommt es zur Entstehung impliziter Prädikationen.

Sowohl in den traditionellen zur Beschreibung der klassischen Sprachen wie Latein oder Griechisch entwickelten Grammatikkonzepten (schon bei Aristoteles) als auch in vielen sprachphilosophischen Arbeiten (GABELENTZ 1868) und modernen linguistischen Sprachtheorien (z.B. CHOMSKY 1965) wird von Subjekt und Prädikat als den zwei konstituierenden Bestandteilen des Satzes ausgegangen. Der Satz wird demnach aufgefasst als eine komplexe Einheit aus einer Substanz, die als begriffliche Vorstellung im Bewusstsein des Sprechenden auf eine außersprachliche Wirklichkeit bezogen ist, und einer Aussage, die über diese Substanz getroffen wird. Angesichts der dichotomischen Auffassung der Satzstrukturen drängt sich die Frage auf, wie dieses Konzept mit den hier aufgeführten infiniten bzw. verblosen Konstruktionen, die keine solche Zweiteilung an der syntaktischen Satzoberfläche aufweisen, in Einklang zu bringen ist und - was vielleicht noch wichtiger ist - ob man sie trotz der fehlenden verbalen Finitheitsträger als Sätze einordnen kann. Bei aller Wichtigkeit von Definitionsansätzen, die in der Fachliteratur zu dem behandelten Themenkomplex vorgeschlagen und stets indioethnisch, also einzelsprachlich-relativ formuliert werden, müssen gewisse universelle Satzparameter erarbeitet werden, die als invariante Merkmale von allgemeiner Relevanz auf andere Sprachen appliziert werden könnten (vgl. auch KoTIN 2007: 195). Die Bindung des Satzes an ein overtes Subjekt und ein overtes Prädikat kann somit nicht als absolutes Kriterium bei der Einordnung sprachlicher Strukturen als Sätze bzw. als Nicht-Sätze gelten. Viel wichtiger ist hier die Existenz einer Prädikation, unabhängig davon, ob diese in formaler Sicht explizit (finit) oder kovert (nichtfinit) realisiert wird. Das soll heißen, dass koverte Prädikationsmarker genauso wie overt realisierte Verben einen Satz zu konstituieren vermögen. Demnach kann zwischen Sätzen mit einer overten, in Form eines finiten Verbs kodierten Prädikation, und Sätzen, die eine koverte, implizite Prädikation enthalten, differenziert werden. Ein solches Herangehen weist auch in methodologischer Sicht Vorteile auf: Es ermöglicht eine holistisch-integrative Erfassung verschiedener Satzstrukturen, ohne dass dabei eine scharfe Trennung zwischen Äußerungen, die sonst einander ähnlich sind, gemacht werden muss, wodurch syntaktische Konstruktionen mit koverten Prädikationsmerkmalen in die empirisch fundierte Forschung mit einbezogen werden können. Grundsätzlich kann man die in dem empirischen Teil ermittelten Typen von koverten Prädikationen nach der Art ihrer 
Etablierung charakterisieren. Die wichtigsten Etablierungsstrategien der koverten Prädikationen lassen sich wie folgt zusammenstellen ${ }^{24}$ :

1) Etablierung der koverten Prädikation über ein Satzglied (subjektoder objektgebundene Prädikation);

2) Etablierung der koverten Prädikation direkt über ein overtes Verb;

3) Mehrfache Etablierung der koverten Prädikation (gleichzeitig durch mehrere Satzglieder, z. B. durch ein Satzsubjekt und ein overtes Verb);

4) Etablierung der koverten Prädikation über ein „Pseudo-Verb“ (vgl. z. B. das to-Element im Polnischen);

5) Etablierung der koverten Prädikation durch Ellipse (Thomas ging ins Kino und Maria ging ins Theater);

6) Etablierung der koverten Prädikation durch besondere Satzstrukturen (Er-und Geschirr spülen?).

Zum Schluss bleibt noch zu fragen, ob Satzentitäten mit koverten Prädikationsmerkmalen (z.B. Partizipien) gegenüber ihren overten Pendants (finiten Verben) - falls diese in einer Struktur vorhanden sind - statusmäßig gleichrangig sind. Das ist nicht der Fall. Vielmehr muss angenommen werden, dass finite Prädikationsmarker den koverten Prädikationsträgern stets übergeordnet sind und das strukturell-semantische Zentrum des Satzes bilden. Demgegenüber bedürfen die meisten infiniten Prädikationsmarker einer (formalen) Einbettung in die Basisproposition, was exemplarisch an den Belegen mit partizipialen Satzmustern gut nachvollziehbar ist. Allerdings ist die Abhängigkeit von der overten Satzprädikation skalar und kann von Fall zu Fall variieren. Es gibt auch Fälle, in denen die koverte Prädikation konstruktionell selbstständig realisiert werden kann (vgl. z. B. die deutschen Beispiele 7-21) und derartige Syntagmen weisen stets eine spezifische syntaktische Struktur (vgl. z.B. das deiktisch-operative Element to bei polnischen Belegen) auf. Welche syntaktisch-distributionellen und semantisch-ontologischen Eigenschaften die einzelnen koverten Satzmuster im Deutschen, aber auch im Polnischen sowie den anderen historischen und modernen germanischen und slawischen Sprachen haben, darf ebenso weiteren Forschungen vorbehalten bleiben wie nicht zuletzt auch die Antwort auf die Frage, wie es überhaupt zur Entstehung von koverten Prädikationsmustern gekommen ist.

24 Diese Typologie erhebt keinen Anspruch auf Vollständigkeit und basiert auf dem für diese Studie zusammengestellten Untersuchungsmaterial. 
Finitheit und Infinitheit

\section{Quellen}

Biblia Tysiąclecia (1971). Poznań/Warszawa.

Das Hildebrandslied (1945). Hrsg. von Georg Baesecke. Halle (S.).

Die Bibel (2006). Einheitsübersetzung. Altes und Neues Testament. Freiburg/Basel/Wien.

Die Gotische Bibel. Der gotische Text und seine griechische Vorlage (72000). Bd. 1. Hrsg. von Wilhelm Streitberg. Heidelberg.

Die lateinisch-althochdeutsche Tatianbilingue Stiftsbibliothek St. Gallen Cod. 56 (1994). Hrsg. von Achim Masser. Göttingen (=Studien zum Althochdeutschen 25).

\section{Literatur}

Ágel, Vilmos / Eichinger, Ludwig M. / EROMs, HANs-Werner et al. (eds.) (2003): Dependenz und Valenz. Ein internationales Handbuch der zeitgenössischen Forschung. Bd. 2: Dependency and Valency. An international Handbook of Contemporary Research. Vol. 2. Berlin.

CHOMsкy, NoAm (1965): Aspects of the Theory of Syntax. Cambridge (Mass.).

CRISTOFARO, SONIA (2007): Deconstructing categories: finiteness in a functionaltypological perspective. In: NikOLAEVA, IrINA (ed.): Finiteness. Theoretical and Empirical Foundations. Oxford, 91-114.

DONHAUSER, KARIN (2007): Zur informationsstrukturellen Annotation sprachhistorischer Texte. In: Sprache und Datenverarbeitung 31(1-2):39-45.

EISENBERG, PETER (1986): Grundriß der deutschen Grammatik. Stuttgart.

- (1998 / $\left.{ }^{3} 2006\right):$ Grundriss der deutschen Grammatik. Das Wort. Stuttgart/Weimar.

ENGEL, UlRICH (1977 / $\left.{ }^{3} 1994\right)$ : Syntax der deutschen Gegenwartssprache. Berlin.

- (1988/ $\left./{ }^{3} 1996\right)$ : Deutsche Grammatik. Heidelberg.

EROMS, HANS-WERnER (2008): Stil und Stilistik. Eine Einführung. Berlin.

FLEISCHER, JÜRG (2006): Zur Methodologie althochdeutscher Syntaxforschung. In: Beiträge zur Geschichte der deutschen Sprache und Literatur 128:25-69.

GABELENTZ, GEORG VON DER (1868): Ideen zu einer vergleichenden Syntax - Wortund Satzstellung. In: Zeitschrift für Völkerpsychologie und Sprachwissenschaft 6: 376-384.

Geist, LuUdmila / BŁaszCZaK, Joanna (2000): Kopulasätze mit den pronominalen Elementen to/eto im Polnischen und Russischen. In: ZAS Papers in Linguistics 16:115-139.

Givón, TAlmy (1990): A Functional-Typological Introduction. Vol. II. Amsterdam. GRZEGORCZYKOWA, RENATA (2004): Wyktady z polskiej sktadni. [Vorlesungen über die polnische Syntax]. Warszawa. 
Gvozdeckaja Natalia Ju. (2002): Drevneanglijskoje poetičeskoje slovo v strukturach teksta. In: GVOZDECKAJA NATALIA Ju. / KRIVUŠIN, IGOR V. (eds.): Rannesrednevekovyj tekst: problemy interpretacii. Ivanovo, 124-151.

Helbig, Gerhard / Buscha, JOACHIM (1972 / $\left.{ }^{10} 1987\right)$ : Deutsche Grammatik. Ein Handbuch für den Ausländerunterricht. Leipzig.

HERINGER, HANS-JÜRgEN (1984): Neues von der Verbszene. In: STICKEL, GERHARD (ed.): Pragmatik in der Grammatik. Jahrbuch des Instituts für Deutsche Sprache. Düsseldorf (=Sprache der Gegenwart 60), 34-64.

JOSEPH, BRIAN D. (1983): The Synchrony and Diachrony of the Balkan Infinitive. Cambridge.

KÖLLER, Wilhelm (2004): Perspektivität und Sprache. Zur Struktur von Objektivierungsformen in Bildern, im Denken und in der Sprache. Berlin/New York.

Kotin, Michail (2007): Die Sprache in statu movendi. Sprachentwicklung zwischen Kontinuität und Wandel. Bd. 2. Heidelberg.

- (2012): Gotisch. Im diachronen und typologischen Vergleich. Heidelberg.

- (2013): Sein. Eine genealogisch-typologische Fallstudie über das Verbum substantivum. (Manuskript)

Nikolaeva, Irina (2007): Introduction. In: Nikolaeva, Irina (ed.): Finiteness. Theoretical and Empirical Foundations. Oxford, 1-19.

PAul, HeRmAnN (1880 / $\left.{ }^{10} 1995\right)$ : Prinzipien der Sprachgeschichte. Tübingen.

Schatte, Christoph, (2011): Zur Gestalt und Semantik von Modalangaben und Modalsätzen. In: Kotin, Miachil / Kotorova, Elizaveta (eds.): Geschichte und Typologie der Sprachsysteme. Heidelberg, 239-244.

SCHÖNHERR, MONIKA (2012): Zur Interdependenz von Wortstellung und Informationsstruktur im Althochdeutschen. In: Sprachwissenschaft 37/2:125-155.

TEubert, WolfGang (1979): Ergänzungen und Angaben beim Substantiv. In: Mitteilungen des Instituts für deutsche Sprache 5:17-26.

ZIFONUn, Gisela et al. (1997): Grammatik der deutschen Sprache. 3 Bde. Berlin/New York. 